\title{
RELATIONSHIPS BETWEEN INTERFACE STRUCTURE AND SUPERPLASTIC DEFORMATION
}

\author{
R. RAJ
}

Department of Materlals Science and Englneering. Bard Hall, Cornell University, Ithaca, NY 14853-1501, U.S.A.

\begin{abstract}
Superplastic flow in polycrystals is achieved through atom by atom transport of matter from interfaces stressed in compression to those in tension. The rate of superplastic flow is therefore controlled by the rate of emission and absorption of atoms at interfaces and by the rate of grain boundary diffusion. The structure of the interface can influence both these processes. In recent years the understanding of the relationship between structure and superplastic behavior has evolved through a multifaceted approach that includes phenomenology, micromechanical modeling, critical experiments, and studies of interface structure in a variety of materials. On this basis, three types of structure dependent superplastlc flow are explored in this paper. In Type $I$, boundary structure is such that the exchange of atoms at the interface is much faster than grain boundary diffusion. In this type boundarles generally have a high $\Sigma$ structure. In Type II, boundaries contain a small amount of fluid which leads to enhanced diffusion at the interface provided that the crystal phase is at least somewhat soluble in the liquid. In Type III the interfaces are predominantly low angle boundaries. Interestingly, the last type produces the fastest rates of superplastic deformation and, therefore, is technologically most significant, but also is the least understood. We speculate that grain boundary dislocations shuttle between interfaces to produce Type III superplasticity.
\end{abstract}

\section{INTRODUCTION}

Superplastic flow is the ability of polycrystalline solids to deform at temperatures considerably below the melting point, in a way that is phenomenologically similar to the flow of glass. Elongations of several thousand percent can be obtalned without flow localization and fracture that characterizes ordinary deformation of crystalline solids. The technological significance of superplastic flow is highlighted by the fact that materlals which are very hard such as ceramics can deform in superplastic flow with ease. Often, superplastic deformation is the only mechanism that can be employed in net-shape forming of hard materlals.

A detailed understanding of superplastic flow develops when the phenomenon is approached from several standpoints that include the mechanics of flow localization In large deformations, modelling of superplastic flow using a materials science approach, and the knowledge of the atomlc structure of grain boundaries. Each is discussed below:

\section{(a) Mechanics of Flow Localization}

From a continuum standpoint, large deformations in a strain rate sensitive material are possible only if the strain rate sensitivity index, $m$, in the flow equation is close to unity. The ability of a material to resist flow localization is explicitly related to the strain rate sensitivity index [1]. The Index is defined by the relationship between the deviatoric (or shear) stress, $\sigma_{e}$, and the deviatoric strain rate, $\varepsilon$, as given below:

$$
\sigma_{e}=B \dot{\varepsilon} \frac{m}{e}
$$


The parameter B In Eq. (1) Includes the effects of temperature and microstructure. The equation assumes that the microstructure remalns constant during deformation. The formability of a material is tled to the value of $\mathrm{m}$ in $\mathrm{Eq}$. (1). In the Ideal case, $\mathrm{mm}$, the materlal behaves like a Newtonian viscous fluld, and is indefinitely deformable. In reality, even smaller values of m lead to ductility that is large enough for most forming operations [2]. Generally, in polycrystals, the flow is assumed to be potentially superplastic if $\mathrm{m}$ lies in the range $0.4<\mathrm{m}<1.0$.

\section{(b) Micromechanistic Modelilng of Superplastic Flow}

The success of superplastic flow emanates from the fact that two classical mechanisms of diffusional creep known as Nabarro-Herring creep [3] and Coble creep [4], lead to an equation of the type given by Eq. (1) where $\mathrm{m}=1$. The mechanism involves transport of atoms from one interface to another either by lattice diffusion ( $\mathrm{N}-\mathrm{H}$ creep), or by boundary diffusion (Coble creep). In fact both transport paths are additive which leads to the following general equation for diffusional creep $[5,6]$ :

$$
\sigma_{e}=\dot{\varepsilon}_{e} \frac{d^{3}}{14 \Omega\left(\delta D_{b}+\pi d D_{v}\right)}
$$

where $\Omega$ is the atomic volume, $d$ is the grain size and $D_{b}$ and $D$ are the boundary and lattice diffusion coefficients. $\delta$ is the diffusional width of the grain boundary. The derivation of Eq. (2) assumes that grain interfaces are 1 deal sources and sinks of atoms, that $1 \mathrm{~s}$, the interface reaction is much faster than the time required for the atoms to diffuse over a distance of about one grain size. Equation (2) assumes that the entire polycrystal deforms by diffusional creep. If this were so then the ideal value of $\mathrm{m}=1$ will be obtained. Indeed, that has been found to be the case for hard materials such as ceramics $[7,8,9]$. But in metals, other deformation mechanisms intervene which have a smaller value of $\mathrm{m}$; this often leads to mixed mode of deformation between diffusional creep for which $\mathrm{m}=1$, and power law creep for which $\mathrm{m} \leq 0.25$. In the mixed mode region, a condition can usually be found where $m=0.5$ so that superplastic behavior is obtained $[10,11]$.

\section{(c) Grain Boundary Structure}

The derivation of Eq. (2) assumes the grain boundary to have very simple properties. It assumes (a) that the boundary provides a path for fast diffusional transport [12], and (b) that the atoms can be absorbed and emitted at the boundary with ease. The fact that the second assumption appears to hold in many materlals Is remarkable; it implies that the growth of a crystal that meets another crystal at a grain boundary can be at least as fast as the growth of free crystal surface from the vapor phase.

This note is concerned with those effects in superplastic flow that are specifically a result of the fact that grain boundaries have structure. The structure can influence superplastic flow in various ways. The transport properties of the interface are likely to be structure dependent. The rate at which atoms can exchange with the crystals may become limited by the structure of the interface. In ceramics the Interface can trap a very thin film of a fluid phase that can enhance diffusion. Another consequence of structure is that the growth and dissolution of crystals can lead to enhanced mobility of the interface; this results in. grain growth that is entirely strain induced. The phenomenon of strain induced grain growth is not only interesting fundamentally, it is also technologically important since the flow stress increases as the third power of the grain size (see Eq. 2); a large Increase in the flow stress can abort a superplastic forming process.

The ideas given above reflect unusual experimental aspects of superplastic behavior that cannot be explained in terms of Eq. (2). They reflect the Importance of the structure of the grain boundary. In order to give form to these ideas we classify the relationship between superplastic flow and the structure of grain boundaries into three types: 
Type I. Here the boundaries are assumed to be high $\Sigma$ boundaries. Typically these will be found in single phase materials when the grain size is greater than a few micrometers.

Type II. Fine-grained polycrystals that contain a few volume percent of a fluid phase in grain interface reglons form an important class of ceramic materials. The presence of even a very small amount of the fluid, less than 1 percent, for example, can have a dramatic effect on the superplastic characteristics of the polycrystals.

Type III. This type is the least understood and potentially of greatest technological importance because it appears to produce very fast rates of deformation. We assume that in this case boundaries are assumed to be predominantly of low angle. Their structure is described as arrays of lattice dislocations. Recent work shows that the population of low angle boundaries in polycrystals increases as the grain size gets smaller [13]; thus Type III may be relevant to nanometer grain size polycrystals.

In the following sections features of each of the three types are discussed with the intention of showing the different ways in which grain boundary structure can lead to distinctly different superplastic behavior. A schematic description of the three types of grain boundaries is given in Fig. 1.

Type I

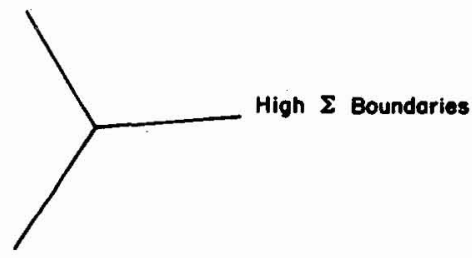

Type II

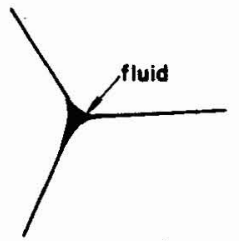

Type III

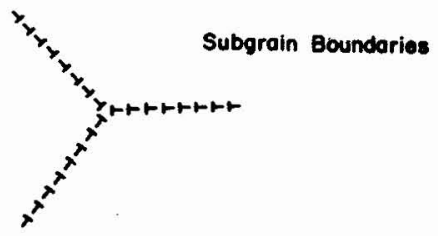

Fig. 1. The three types of superplastic flow are based upon differences in grain boundary structure. In Type I boundaries are normal, high $\Sigma$ boundaries. They occur most commonly in single phase polycrystals having a grain size of a few $\mu \mathrm{m}$. In Type II boundarles contain a thin film of a fluld phase; this is often the case in ceramic materials. In Type III the boundaries are predominantly low angle or subgrain boundarles. 


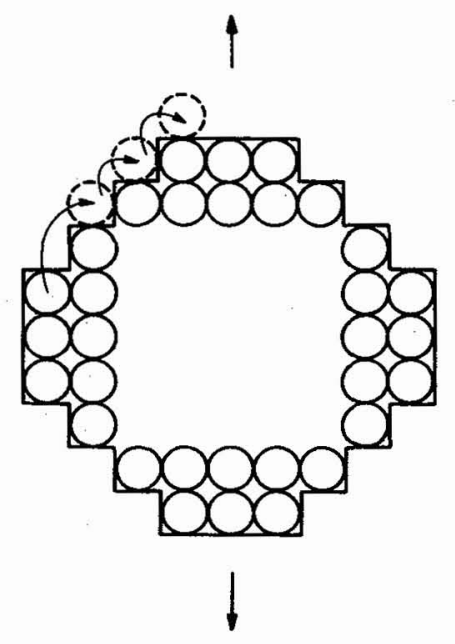

Fig. 2. A schematic showing how a crystal grain can change its shape by the diffusion of atoms along its surface. A similar process can occur in polycrystals except that atoms move along interfaces.

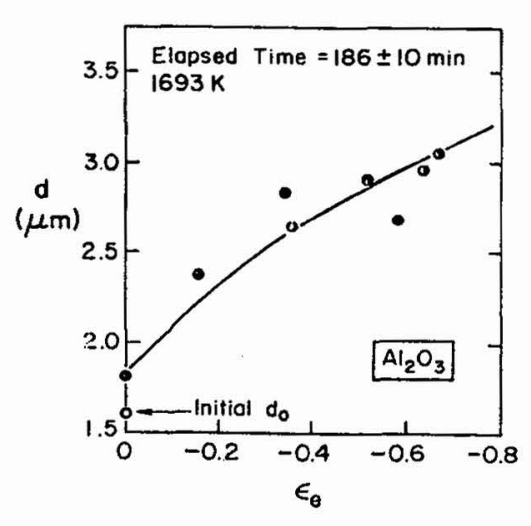

Fig. 3. Strain Induced grain growth In alumina during superplastic deformation. The open point is the virgin grain size. The full points have the same time and temperature history but represent varying amounts of applied strain; the $\varepsilon=0$ specimen was annealed without deformation while the other specimens were deformed at increas ing strain rates.

\section{TYPE I: Superplastic Flow by Nabarro-Herring/Coble Creep}

In his classical derivation of diffusional creep Herring [3] assumed that grain boundaries are perfect sources and sink of vacancies. The mechanism is illustrated schematically in Fig. 2 by means of a single grain that changes its shape by diffusion of atoms along the surface. In a polycrystal all the grains deform in self-similar fashion so that the external deformation is the same as the deformation of one grain. The interesting point about this mechanism is that the rate of deformation can be related explicitly to the kinetics of atom transport and to the kinetics of crystal growth and dissolution at grain boundaries. Just as crystal growth and solid state transformation kinetics can be interface limited or diffusion limited [14], diffusional creep can also be limited either by the rate at which atoms are removed from or attached to the crystals at the grain boundaries, or by the rate at which the atoms are transported over a distance of about one grain size. Herring [3] assumed that the transport of atoms was the limiting factor. Coble [4] also made the same assumption but he considered the case where transport occurred predominantly through the grain boundaries, whereas Herring had addressed diffusion through the crystal lattice. Both diffusion paths are in fact additive which leads to the result given in Eq. (2).

The assumption inherent in Eq. (2), that creep is diffusion rather than interface reaction limited, can be experimentally confirmed by studying the grain size dependence in the equation. Interface limited creep would give a linear grain size dependence [15] Instead of the square or the cubic dependence obtained for the 
diffusion limited case. Nearly all experimental data, obtained on a varlety of materials, shows a cubic grain size dependence (fast strain rates of deformation require a grain size of a few micrometers or smaller; at these grain sizes the boundary diffusion term in Eq. (2) is dominant which prescribes a cubic grain size dependence of flow stress). Clear evidence of linear grain size dependence has not yet been obtained, leading to the inference that grain boundarles do not create a barrier to the growth and dissolution of crystals. In one sense this is not a surprising result. Since boundaries have structure, the diffusion of atoms along the interface probably involves jumps between ledge sites, which is also mechanism that underlies the growth and dissolution of the crystal; diffusion over the distance of several atom spacings, therefore, would be necessarily slower than the interface reaction.

The fact that interfaces have definite structure is belleved to lead to the unusual phenomenon of strain induced grain growth in superplastic deformation of single phase materials. The results for alumina [7] are shown in Fig. 3; the open circle shows the initial grain size and the full circles show the grain size as a function of the applied strain. The full circles represent specimens which had experfenced the same time and temperature history but different strain (and strain rate) history; for example, the point at zero strain was simply annealed without deformation, and the other points were deformed for the same time and at the same temperature but at different strain rates. Strain induced grain growth leads to strain hardening in a constant strain rate experiment because the flow stress increases as the third power of the grain size; this is shown in Fig. 4 for the case of a superplastic aluminum alloy [16].

In summary, Type I superplasticity is 1 imited by the transport properties of grain interfaces. Single phase polycrystals that contain mostly high $\Sigma$ boundarles follow this behavior. The unusual effect of Interface structure is that it leads to strain induced grain growth; this phenomenon is most probably tied to the atomistic mechanism involved in superplastic deformation where atoms are transported in order to change the shape of the grains by a crystal-growth-and-dissolution-like process. The structure in the boundary can produce asymmetric growth in the crystals that meet at an interface; as a result some crystals can grow at the expense of their neighbors leading to strain induced grain growth. A definitive study of this mechanism has not yet been carried out.

\section{TYPE II: Superplast1c F1ow Enhanced by a Fluid Phase}

Impurity segregation in grain boundaries can create a liquid phase if the segregants form a low temperature eutectic with the crystalline phase. In ceramics, the presence of silica at interfaces is a common cause of a liquid eutectic phase. The residual silica can arise from additives that are used to promote sintering (example: silicon nitride), or from incomplete crystallization of the glass in glass ceramic materials (example: $\beta$-spodumene), or from unintentional absorption of silica during powder processing and sintering since silica is a common impurity in the environment and, especially, in the insulation materials used in high temperature furnaces. The presence of a thin fluid layer of a silicon-oxynitride glass in silicon nitride has a dramatic effect on the creep rate [17]. The data in Fig. 5 show that silicon-nitride that is processed with a glass additive deforms much faster than the reaction bonded material which is free from silica. In another study [18] a comparison between the creep of aggregates of potassium chloride that were saturated in one case with water and in another case with an alcohol, demonstrated that creep enhancement is not possible unless the crystal is soluble in the fluid [19].

A mechanistic understanding of how the liquid phase enhances creep is not entirely understood, principally because the atomistic details of the structure are not clear. High resolution microscopy of interfaces in silicon-nitride show that the glass resides at the triple junctions while a thin layer, approximately 1 to 2 nm thick, is trapped at the two grain junctions [20]; this result agrees with thermodynamic analysis that is based upon interface free energy arguments [21]. A simple view of liquid phase enhanced creep is that the fluid enhances the rate of diffusional transport through the interface; since the thickness of this layer is believed to be Independent of the total volume fraction of the glass we infer that only a small amount of the liquid is needed to promote creep enhancement; although 


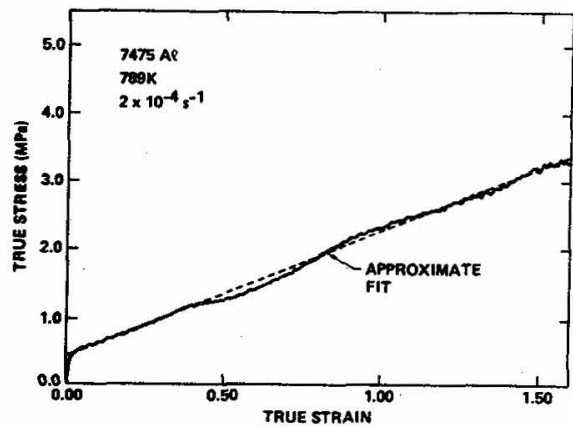

Fig. 4. The increase in flow stress with strain in aluminum as a result of strain induced grain growth. (The flow stress increases as the third power of the grain size.) Courtesy A.K. Ghosh.

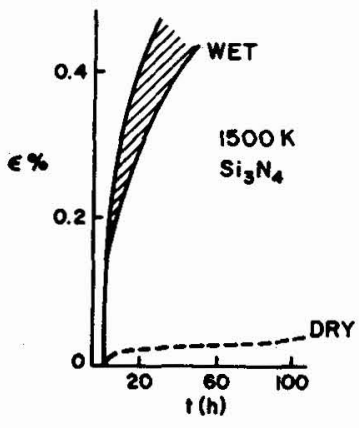

Fig. 5. The influence of approximately 1 to $5 \%$ liquid phase on the creep rate of silicon-nitride [17].

definitive studies of this point have not been done, in general the data support this prediction.

The details of the interface structure become important when one considers the distribution of normal traction at the interface, which is the driving force for stress Induced diffusion, and the diffusion path for atoms through the fluid layer at the interface. The dichotomy arises because we require the interface to support a gradient in normal traction, while a continuous fluid film, that is necessary for enhanced diffusion, cannot support a pressure gradient. In one proposal [22] the interface is assumed to have an island structure where solid-tosolid contacts support traction gradients and the interpenetrating fluid provides a path for fast diffusion (see Fig. 6). The matter remains unresolved. An important phenomenological difference between Type I and Type II behavior is that strain induced grain growth that occurs in Type $I$ is not seen in Type II superplasticity [9]; the explanation should lie in the difference in the structure of the interface. The other difference between the two cases is that interface Iimited creep (with linear grain size dependence of the flow stress) definitely occurs in Type II [22] but has yet to be unambiguously reported in Type I superplasticity.

The flow equation for Type II superplasticlty has been derived in Refs. 19 and 22 by Invoking the mechanical work argument that equates the external work done to the energy dissipated in diffusional transport. Here we give the result for the diffusion limited case [19]:

$$
\sigma_{e}=\dot{\varepsilon}_{e} \frac{n d^{3}}{2.3 \bar{c} \sigma \Omega}
$$

where $\bar{c}$ is the molar solubility of the crystal in the fluid, $\eta$ is the viscosity of the grain boundary phase, $\Omega$ is the atomic volume, $d$ is the grain size, and $\alpha$ is a factor that depends on the structure of the grain boundaries. Note that the equation does not depend on the volume fraction of the liquid phase but it does depend on the viscosity. The linear relation between stress and strain rate, and the cubic dependence on grain size have been unambiguously established experimentally [9] In a model glass ceramic material. A picture showing superplastic deformation in a specimen is reproduced in Fig. 7. 


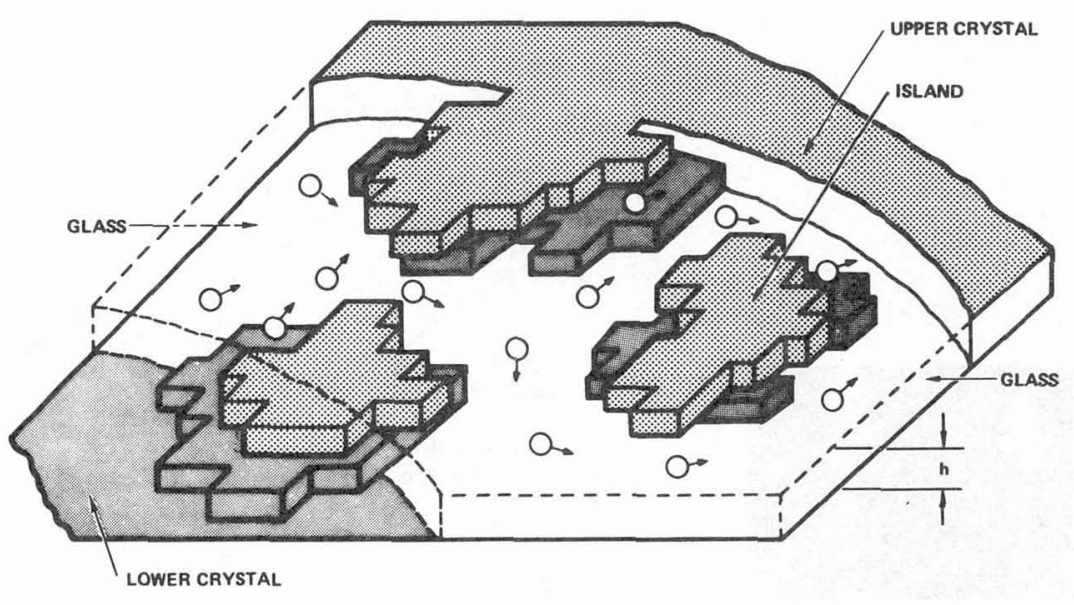

Fig. 6. A proposed island structure of the interface in polycrystals where the fluid phase leads to enhanced rates of deformation. The solid-solid contacts can support the traction gradients while the topologically interconnected fluid layer provides a path for enhanced diffusion [22].

It is very interesting to note in Eq. (3) that the viscosity of a ceramic polycrystal is directly proportional to the viscosity of the glass phase even when the glass is present in sma11 amounts. Presence of dopants can change the viscosity of the glass by several orders of magnitudes, and $1 \mathrm{~s}$, therefore, an $\mathrm{im}-$ portant processing variable. The results in Fig. 8 show the wide range of strain rates that are obtained in $\beta$-spodumene glass ceramics simply by changing the chemistry of the intergranular glass. The graph shows the grain size normalized strain rate plotted against the flow stress. The linear slope for each data set shows adherence to Eq. (3), but the change in the strain-rate intercept is the result of the composition of the intergranular glass. As the glass becomes increasingly pure sllica, the strain rate decreases; the change spans several orders of magnitude in strain rate.

In summary, Type II superplasticity is produced by the presence of a very thin layer of a fluid phase at grain interfaces. The film enhances diffusion but in contrast to Type I superplasticity, strain induced grain growth is not observed. Also, Type II superplasticity shows evidence of interface reaction controlled deformation which has not yet been seen unambiguously in Type I superplasticity. These differences reflect the difference in the atomic structure of interfaces with and without a fluld phase, but are not well understood at this time.

\section{TYPE III: Sub-Grain Superplasticity}

The diffusional mechanisms of superplasticity described as Type I and Type II lead to a strain rate sensitivity, $m$, equal to unity. The definition for $m$ is given in Eq. (1). In the Introduction it was discussed that superplasticity is possible even when $m$ lies in the range $0.4<\mathrm{m}<1.0$. In sub-grain superplasticity $m$ is approximately equal to 0.5 . This case of superplasticlty has been much less studied than the previous two. The mechanism is not well understood and features of this mechanism described here are like1y to be controversial. In-depth studies 

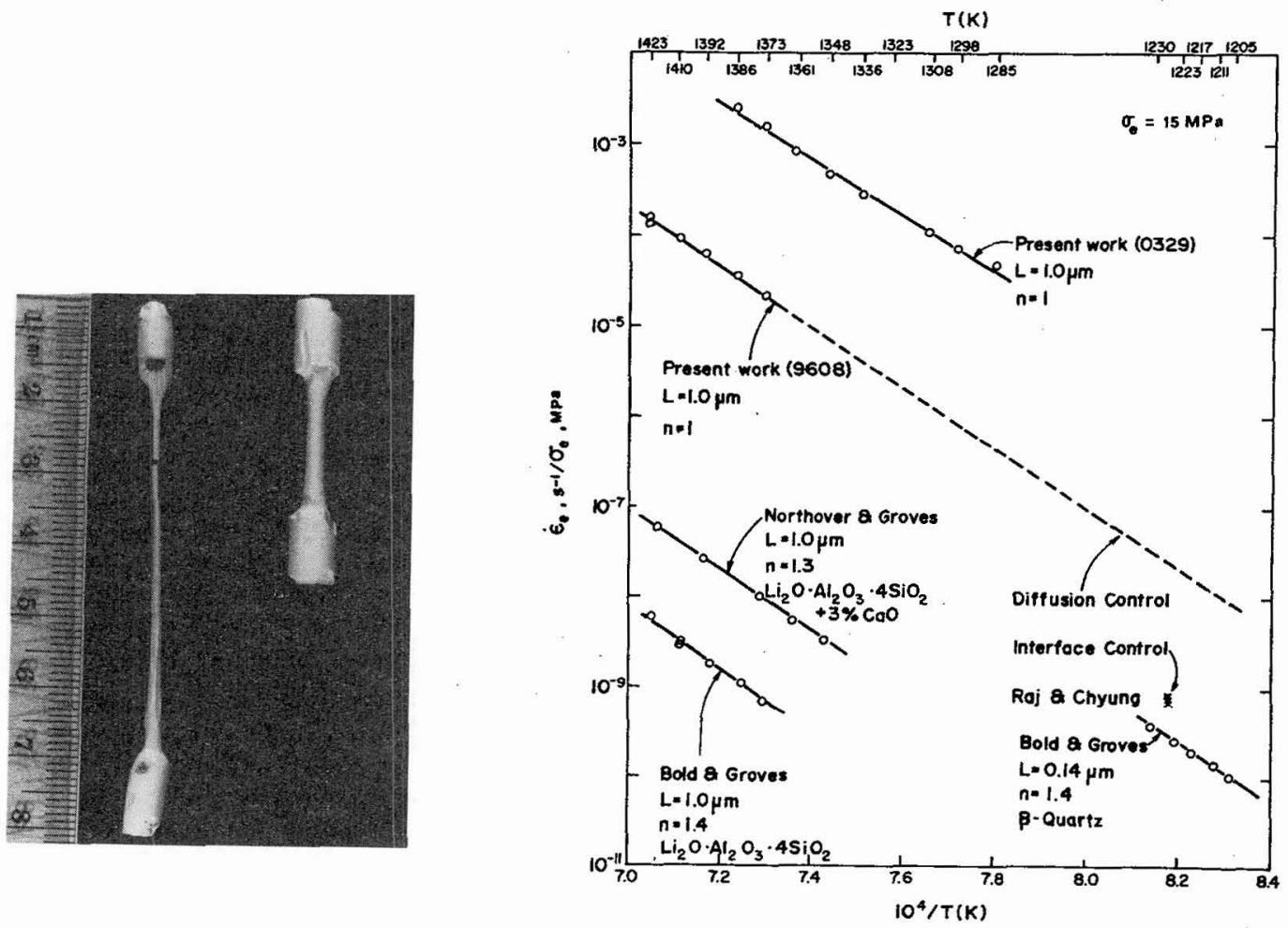

Fig. 7. Superplastic deformation of a ceramic material containing less than $5 \%$ residual glass [9].

Fig. 8. Effect of the composition of the segregated glass phase on the strain-rate of polycrystals of the same crystalline material. The linear slope between grain-size compensated strain rate and the flow stress is in agreement with Eq. (3) $[9]$.

are needed particularly because this type of superplasticity leads to the highest rates of deformation and, therefore, is technologically significant. The special features of Type III superplasticity are now discussed.

Measurement of the strain rate sensitivity and the grain size dependence of the flow stress can distinguish between the diffusional and the dislocation nechanisms of deformation. As discussed in the previous sections the diffusional mechanism requires a grain size dependence such that the flow stress increases rapidly with increasing grain size. In the dislocation mechanism the flow stress is either independent of the grain size or decreases with increasing grain size. The $m$ value is not an unambiguous indicator of a distinction between the dislocation and diffusional mechanisms. While $m=1$ necessarily implies diffusional creep, $m<$ can be a result of either diffusional or dislocation creep.

In at least two ceramic materials and one metal, evidence of subgrain superplasticlty has been obtained. In magnesium aluminate spinel $\mathrm{m}=0.5$ was obtained and the flow stress was found to drop with increasing grain size [23]. In zirconia, $m=0.5$ was measured and dominant mechanism was ascertained to be dislocation creep [24]. In specially processed aluminum alloys $m=0.5$ was measured at very high strain rates [25]; here, two very interesting observations were made with regard to the grain structure: first, the grain size depended on the deformation parameters 
(strain rate and temperature) rather than on the Initial grain size of the polycrystal, and second, the grain boundaries were generally low angle boundaries with a misorientation of between one and five degrees. In aluminum alloys the subgrain size was approximately $1 \mu \mathrm{m}$, In the spinel the subgrain size varied from 10 to 15 $\mu \mathrm{m}$, while in zirconia it is not yet confirmed whether or not the interfaces were low angle boundarles.

Assuming that the subgrain boundaries in Type III superplasticity consist of arrays of lattice dislocations, a model has been proposed [25] where deformation accrues by the transfer of dislocations from one boundary to another via the crystal lattice, as shown schematically in Fig. 9. In the steady state the emission rate of the dislocations, w111 be equal to the arrival rate of dislocations at the adjacent interface; both w111 be equal to the ratio of the dislocation velocity through the lattice divided by the subgrain size, which we call $\mathrm{d}^{\prime}$. If It is assumed that the dislocation velocity is related to the applied shear stress by a linear coefficient of friction $\mu$, then it can be shown [25] that:

$$
\sigma_{e}=\frac{\dot{\varepsilon}_{\mathrm{e}} \mathrm{d}^{\prime}}{\Delta \theta} \cdot \frac{\mathrm{kT}}{2 \mu \Omega}
$$

where $\Delta \theta$ is the misorientation at the boundaries and $\Omega$ is the atomic volume. At a fixed value of the subgrain size, d', the equation predicts a linear relation between stress and strain rate. Actually, experience shows that the subgrain size decreases with increasing strain rate, which means that the flow stress will increase less rapidly with strain rate than suggested by the linear relationship, leading to a strain rate sensitivity of less than one. Eq. [4] is a first step in understanding this type of superplastic flow, but it does ink macroscopic behavior deformation behavior to the structure of interfaces.

In summary, Type III superplasticity is believed to be a result of a polycrystal that contains predominantly small angle grain boundaries. It is the least understood of the three types discussed in the paper but is technologically most significant because it produces very high strain rates. Further work should emphasize this case of superplastic deformation.

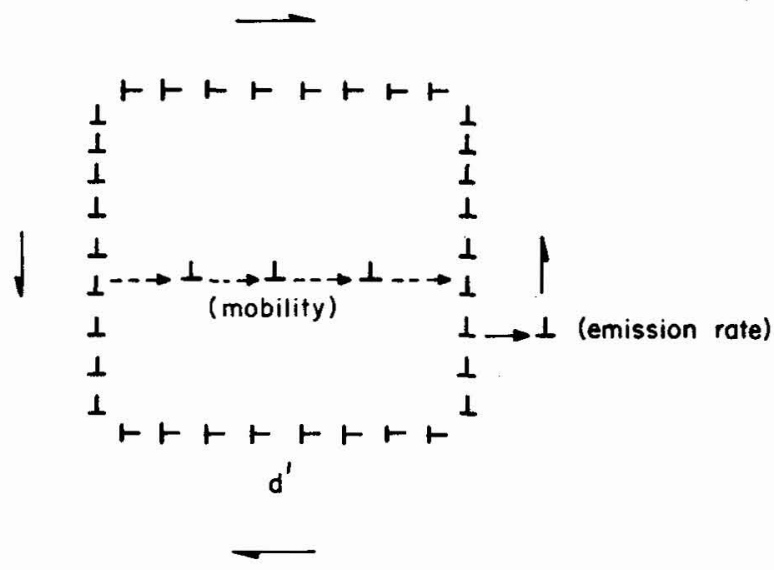

Fig. 9. A schematic explaining the mechanism of Type III superplasticity. The grain boundaries are assumed to be low angle boundaries and deformation occurs by emission and absorption of dislocations between the boundaries. The dislocations travel through the crystal lattice. 


\section{ACKNOWLEDGMENTS}

The work presented here is derived predominantly from Cornell doctoral dissertations by J. G. Wang, K. R. Venkatachari, and P. C. Panda. Their research was funded by the Department of Energy under Grant No. DE-AC02-77ER4386 with partial support from the National Science Foundation through the Materials Science Center at Cornell University.

\section{REFERENCES}

1. E.W. Hart, Acta Metal1. 15, 351 (1967).

2. A.K. Ghosh and R.A. Ayers, Meta11. Trans. 7A [10] 1589-91 (1976).

3. C. Herring, J. App1. Phys., 82, 87-93 (195i).

4. R.L. Coble, J. App1. Phys., $\overline{34}$ [6] 1679-84 (1963).

5. M.F. Ashby and R.A. Verral1, Acta Metal1., 21149 (1973).

6. R. Raj and M.F. Ashby, Meta11. Trans. 2A 1113 (1971).

7. K.R. Venkatachari and R. Raj, J. Am. Ceram. Soc. 69 [2] 135-138 (1986).

8. C. Carry and A. Mocellin, ibid. Ref. 16, pp. 16.1-16.19.

9. J.-G. Wang and R. Raj, J. Am. Ceram. Soc. 67 [6] 399-409 (1984).

10. R. Raj and A.K. Ghosh, Acta Metall., 29 28 $\overline{3-292}$ (1981).

11. A.K. Ghosh and R. Raj, Acta Metall., $\frac{29}{29} 607-616$ (1981).

12. R.E. Hoffman and D. Turnbull, J. App1. Phys. 22634 (1951).

13. T. Watanabe, this volume.

14. J.W. Christian, in The Theory of Transformations in Metals and Alloys, 1st ed., 1965, Pergamon Press, Chaps. X, XI.

15. M.F. Ashby, Scripta Metall. 3837 (1969).

16. A.K. Ghosh and R. Raj, "The Evolution of Grain Size Distribution during Superplastic Deformation," Superplasticity, ed. B. Baudelet and M. Suery, Proceedings of an International Conference, Sept. 1985. Editions du Centre Nationale de la Recherche Scientifique, Paris, pp. 11.1-11.19.

17. R. Osborne, Proc. British Ceram. Soc., vol. 25 (1975)

18. G.M. Pharr and M.F. Ashby, Acta Meta11., 31, 129-138 (1983).

19. R. Raj, J. Geophys. Res., vol. 87, No. B6, 4731-4739 (1982).

20. D.R. Clarke and G. Thomas, J. Am. Ceram. Soc. 60 [11-12] 491-95 (1977).

21. R. Raj, J. Am. Ceram. Soc. 64 [5] 245-248 (1981).

22. R. Raj and C.K. Chyung, Acta Metall. 29, 159-166 (1981).

23. P.C. Panda and R. Raj, J. Am. Ceram. Soc. 68 [10] 522-29 (1985).

24. P.C. Panda, J. Wang and R. Raj, "Sinter Forging Characteristics of Fine Grained Zirconia," to appear in J. Am. Ceram. Soc. (1988).

25. R. Raj, A.K. Ghosh and C. Gandhi, "Subgrain Superplasticity," unpublished work (1985).

W. Gust The coble creep rate is determined by the grain boundary diffusivity. How can we understand in terms of the boundary structure that the diffusivity of grain boundaries under such a high creep rate is similar to the diffusivity of completely relaxed boundaries (stationary grain boundaries)?

R. Raj I believe you are asking if diffusion at grain boundaries can become non linear at fast rates of diffusion, or that sliding boundaries may show an enhancement in the rate of grain boundary diffusion. Unfortumately I do not know of any investigation where superplastic behavior has been studied as a function of temperature. 